anti-Stokes lines tend to meet each other at some finite value, while from the theory we should expect the intensities of the Stokes and the anti-Stokes lines to become large at high temperatures and to tend to meet each other at infinite intensity. (4) The ratio of the intensities of the Stokes and anti-Stokes lines, at all temperatures, is in agreement with the result expected from Placzek's theory.

The polarizability of a molecule may be expanded as a series

$L(q)=L_{0}+\sum_{j}\left(\frac{\partial L}{\partial q_{j}}\right)_{0} q_{j}+\frac{1}{2} \sum_{j k}\left(\frac{\partial^{2} L}{\partial q_{j} \partial q_{k}}\right)_{\theta} q_{j} q_{k}+\ldots$, where the suffix 0 refers to the equilibrium configuration, $q_{j}, q_{k}$, etc., are the various normal $\mathrm{co}^{-}$ ordinates of the molecule, and a particular set of values of $q_{j}, q_{k}$, etc., define a configuration $q$ of the molecule. The conclusions from Placzek's theory are arrived at by taking the factor $\left(\frac{\partial L}{\partial q_{j}}\right)_{0}$ as constant at various temperatures. Taylor's expansion is valid only in the close vicinity of the equilibrium configuration. But as the temperature is increased, the amplitudes become larger, and we can no longer assume that Taylor's expansion, which is taken in the neighbourhood of the equilibrium configuration, is valid. Therefore, $\left(\frac{\partial L}{\partial q_{j}}\right)_{0}$ cannot be regarded as constant. It will decrease rapidly with the increase of temperature. The observed results can be explained on this basis.

A detailed explanation will be published elsewhere.

Andhra University,

K. VENKATESWARLU

Waltair, S. India.

Nov. 12.

\section{Structure of Starch and Cellulose}

Although the characteristic features of the chemistry of starch and cellulose are satisfactorily represented by the commonly accepted structures involving chains of glucopyranosido residues, which are unbranched in cellulose and amylose, but branched in amylopectin, certain difficulties remain to be explained. These include, for example, the exact nature of the relationship between the amylose and the amylopectin components of the starches, and the reasons for the different behaviour of cellulose on methylation in the presence and in the absence of oxygen respectively.

In an attempt to overcome very real difficulties of this kind, Pacsu and Hiller ${ }^{1}$ have recently advocated a novel structure for these polysaccharides based on the assumed occurrence of a small proportion of non-cyclic hemiacetal linkages. This is a modification of the Tollens acetal structure for cellulose, and the essential feature of it as applied to starch is shown in (I), which depicts a maltose residue in a hemiacetal form serving as a link between long chains of $1: 4$ linked glucopyranosido residues. The glucose residue marked with an asterisk is an end-group, and Pacsu and Hiller apparently postulate a sufficient number of these cross-linkages to account for the number of end-groups as determined by the methylation method. It is not possible, therefore, to decide on the results of methylation experiments alone whether or not such a formula is valid for a starch.

Other methods of approach to the problem of end-group determination have recently been de- veloped, and by application of one of these we have obtained evidence from which it appears that the type of linkage suggested by Pacsu and Hiller does not occur in starches. Inspection of $(\mathrm{I})$ reveals that the presence of hemiacetal linkages increases the number of contiguous hydroxyl groups at which oxidative attack by the periodate ion is possible, with the result that from the structure shown in (I) three molecules of formic acid would be formed for every terminal glucose residue $\left(G^{*}\right)$. Actually, on the basis of the much more complicated detailed formula depicted by these authors, considerably more than three molecules of formic acid per end-group would be liberated, with the simultaneous formation of a large number of fragments of smaller molecular weight.

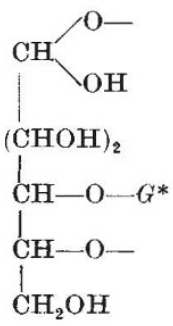

(I)

It has been found, however, by use of methods elaborated recently for the quantitative liberation and estimation of formic acid in periodate oxidations of polymeric glycosides ${ }^{2}$, that starches yield only one molecule of formic acid per end-group. This result is in agreement with the expected value if amylopectin possesses the 'laminated' structure ${ }^{3}$, but is incompatible with Pacsu and Hiller's formula.

In other respects also the newly proposed structure appears to be incapable of reconciliation with experimental observations. For example, investigations on the kinetics of the 'disaggregation' of starch have shown that the bonds broken in the reaction have the stability of glycoside links towards acid, and require no novel type of bonding to explain their properties ${ }^{4}$. Furthermore, it is difficult to see how the results of enzymic hydrolysis and dextrin formation could be explained by structures involving hemiacetal linkages of the type shown in (I).

\section{T. G. Halsali \\ E. L. Hirst \\ J. K. N. JONES}

University, Manchester.

Nov. 29

${ }^{1}$ Pacsu, E., and Hiller, L. A., Text. Res. J., 16, 243 (1946)

2 Jackson, E. I., and Hudson, C. S., J. Amer. Chem. Soc., 58, 378 (1936) ; 59, 994 (1937). Brown, F., Dunstan, Sonia, Halsall. T. G., Hirst, E. L., and Jones, J. K. N̈., Nature, 156, 785 (1945). s Haworth, W. N., Hirst, E. L., and Isherwood, F. W., J. Chem. Soc., 577 (1937).

' Bawn, C. E. H., Hirst, E. L., and Young, G. T., Trans. Faraday Soc., 36, $880(1940)$.

\section{Effects of Organic Arsenic Compounds on Tissue Enzymes and Proteins and on Tissue Metabolism}

IN connexion with recent interesting reports, both in Great Britain and in the United States, on the relationship between organic arsenicals and enzymes containing thiol groups, we would like to take the opportunity of directing attention to some early. observations of our own, incorporated in a report to the Medical Research Council in November 1940. 